Europhys. Lett., 63 (1), pp. 104-110 (2003)

\title{
Origin of temperature dependence in tunneling magnetoresistance
}

\author{
J. J. Ákerman ${ }^{1,2}$, I. V. Roshchin ${ }^{1}$, J. M. Slaughter ${ }^{2}$, \\ R. W. DAve ${ }^{2}$ and I. K. Schuller ${ }^{1}$ \\ 1 Physics Department 0319, University of California San Diego \\ La Jolla, CA 92093-0319, USA \\ 2 Motorola Labs, Physical Sciences Research Laboratories - Tempe, AZ 85284, USA
}

(received 4 November 2002; accepted in final form 25 April 2003)

PACS. 73.40.Gk - Tunneling.

PACS. 73.40.Rw - Metal-insulator-metal structures.

PACS. 75.70.Pa - Giant magnetoresistance.

\begin{abstract}
We present detailed measurements of the differential resistance $(\mathrm{d} V / \mathrm{d} I)$ of stateof-the-art $\mathrm{FM} / \mathrm{AlO}_{x} / \mathrm{FM}$ magnetic tunnel junctions (MTJ) as a function of applied bias and temperature. Temperature effects are particularly significant in physical quantities involving narrow features such as those at low-voltage bias. We show that the temperature evolution of the tunneling characteristics and, in particular, the pronounced rounding of the $\mathrm{d} V / \mathrm{d} I$ curves with increasing temperature can be well explained by thermal smearing of the tunneling electron energy distribution.
\end{abstract}

Tunneling magnetoresistance [1] (TMR) in magnetic tunnel junctions (MTJ) continues to receive increasing attention [2], since high TMR values [3,4] allow for applications such as magnetic random access memory [5] (MRAM) and magnetic read-heads [6]. Although the low-temperature TMR can reach the optimum values expected from Julliere's model [1], even high-quality MTJs suffer a significant loss of TMR with increasing temperature. It was suggested that emission of surface magnons by tunneling electrons lead to extra current channels through the tunneling barrier. The contribution from these channels grows with the number of thermally excited magnons, more so for the antiparallel (AP) configuration, with the consequent overall increase in conductivity and corresponding loss in TMR with increasing temperature $[7,8]$. In a different approach, Julliere's model was extended [9] to include both a temperature-dependent surface spin polarization, and a spin-independent current channel, assumed to arise from hopping within the barrier [10-12]. Other proposed mechanisms include barrier defect and/or magnetic impurity scattering [13-16] and band structure effects [17]. Although thermal smearing is known to dominate the temperature dependence of superconducting tunneling [18], the same mechanism has been largely ignored in MTJs, based on the conclusions in Simmons' original theoretical work [19] where thermal effects are found to be very small for elastic normal-state tunneling through an ideal trapezoidal barrier.

In this work, we show that thermal smearing plays a much more important role in determining the temperature dependence of TMR than previously believed. Inclusion of thermal smearing in the analysis of experimental data enables us to explain a number of experimental observations, such as the much stronger $T$-dependence of TMR at zero bias compared to finite 
bias, the increased rounding of both the zero-bias anomaly as well as any other particular feature in the tunneling data, and the known correlation between a strong bias dependence and a strong temperature dependence of TMR. In the light of the above, any attempt to attribute the temperature dependence of TMR to a particular mechanism, without first determining the effect of thermal smearing, appears inadequate. We must stress that thermal smearing is always present, independently of any other temperature-dependent mechanism.

Details of the sample fabrication are reported in ref. [20]. Briefly, the bottom-pinned MTJ material uses an IrMn exchange layer, a NiFeCo/CoFe bilayer for the bottom magnetic electrode, and $\mathrm{NiFeCo}$ alloy for the top magnetic electrode. The $\mathrm{AlO}_{x}$ tunnel barrier is formed by depositing approximately $10 \AA$ of $\mathrm{Al}$ on the bottom electrode followed by oxidation in an RF-produced oxygen plasma. Two different oxidation times of $60 \mathrm{~s}$ and $240 \mathrm{~s}$ are chosen to form junctions with a final resistance-area products (RA) of approximately $1.3 \mathrm{k} \Omega \mu \mathrm{m}^{2}$ (sample A) and $24 \mathrm{k} \Omega \mu \mathrm{m}^{2}$ (sample B), respectively. The wafer is annealed at $250^{\circ} \mathrm{C}$ to improve the tunnel barrier and then patterned to a bit size of $4 \times 4 \mu \mathrm{m}^{2}$ by standard lithographic techniques. The wafer is diced and the die is mounted in a ceramic package and bonded with gold wires. Although a standard four-probe configuration was used to connect to the package, there is some small additional series resistance due to the thin-film interconnects within the die.

Figure 1a-d shows the experimental tunneling curves (dotted and dashed lines) at different temperatures for the two representative samples in the antiparallel (AP) and parallel (P) state, respectively. As $T$ increases, $\mathrm{d} V / \mathrm{d} I$ decreases at all bias levels, confirming that tunneling is the predominant conduction mechanism [21]. Both samples exhibit a strong zero-bias anomaly in both states that is gradually reduced with increasing temperature. While the resistance maximum stays close to zero bias at all temperatures in fig. 1a-c, a noticeable shift of $\mathrm{d} V / \mathrm{d} I_{\max }(\mathrm{P})$ to positive bias appears in the $\mathrm{P}$ state of sample $\mathrm{B}$ with increasing temperature (fig. 1d). Other features, most apparent in the $\mathrm{P}$ state of each sample, also appear more rounded with increasing $T$.

Rounding of the $\mathrm{d} V / \mathrm{d} I$ curves with increasing $T$ is more noticeable in the region where the curves have features of width smaller than or comparable to the temperature. In our samples, most such features are present at low bias (below $0.1 \mathrm{~V}$ ), thus the temperature dependence is more pronounced there. The increased rounding with increasing $T$ strongly suggests that thermal smearing plays an important role in the temperature dependence.

Thermal smearing appears via the Fermi distribution function $f$ in the calculation of the tunneling current:

$$
I=\int_{-\infty}^{+\infty} \mathrm{M}(E-V, E) N_{\mathrm{L}}(E-V) N_{\mathrm{R}}(E)[f(E-V)-f(E)] \mathrm{d} E,
$$

where $\mathrm{M}$ is the tunneling matrix element and $N_{\mathrm{L}}$ and $N_{\mathrm{R}}$ are the electron densities of states on the left and the right sides of the junction, respectively [22]. By taking the derivative of eq. (1), we obtain the differential conductance,

$$
\begin{aligned}
\left.\frac{\mathrm{d} I}{\mathrm{~d} V}\right|_{T}= & \left.\int_{-\infty}^{+\infty} \mathrm{M}(E-V, E) N_{\mathrm{L}}(E-V) N_{\mathrm{R}}(E) \frac{\mathrm{d} f(E-V)}{\mathrm{d} V}\right|_{T} \mathrm{~d} E+ \\
& +\left.\int_{-\infty}^{+\infty} \frac{\mathrm{d}}{\mathrm{d} V}\left(\mathrm{M}(E-V, E) N_{\mathrm{L}}(E-V) N_{\mathrm{R}}(E)\right)[f(E-V)-f(E)]\right|_{T} \mathrm{~d} E \\
= & \left.\int_{-\infty}^{+\infty} A(E-V, E) \frac{\mathrm{d} f(E-V)}{\mathrm{d} V}\right|_{T} \mathrm{~d} E+ \\
& +\left.\int_{-\infty}^{+\infty} B(E-V, E)[f(E-V)-f(E)]\right|_{T} \mathrm{~d} E
\end{aligned}
$$




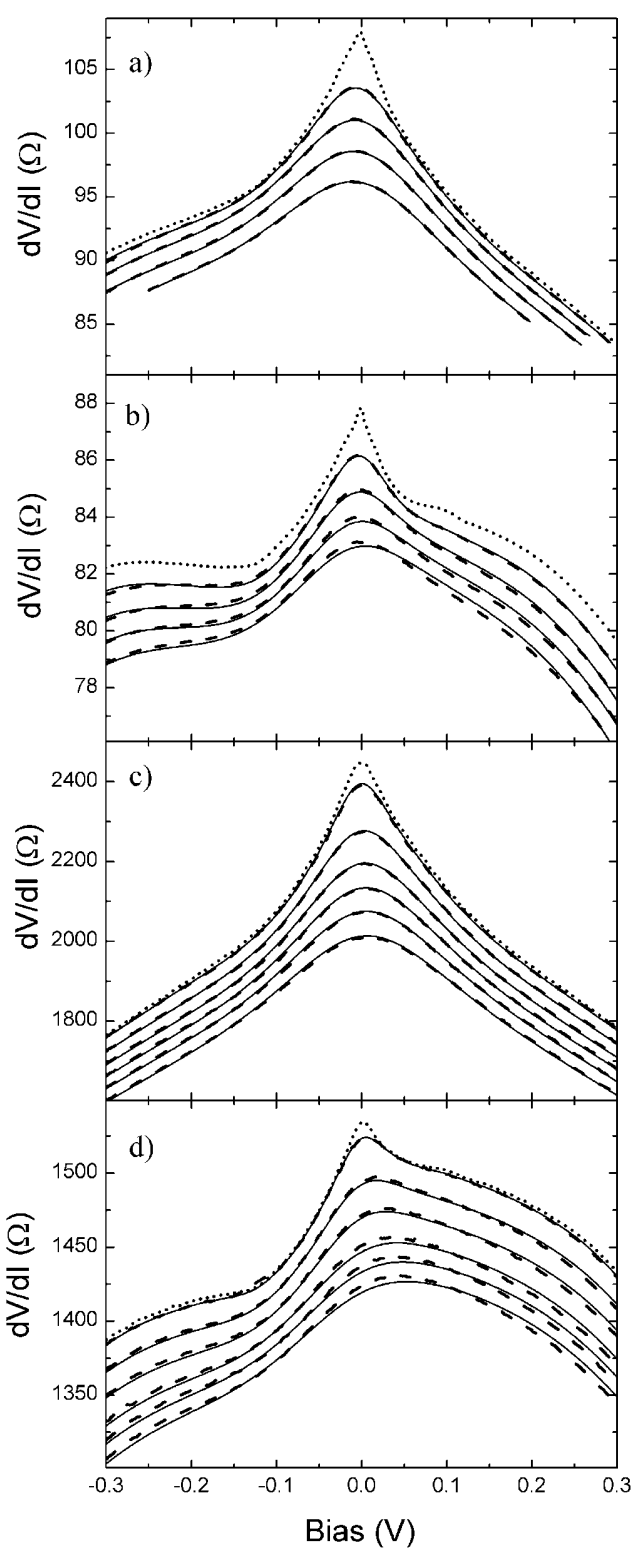

Fig. 1

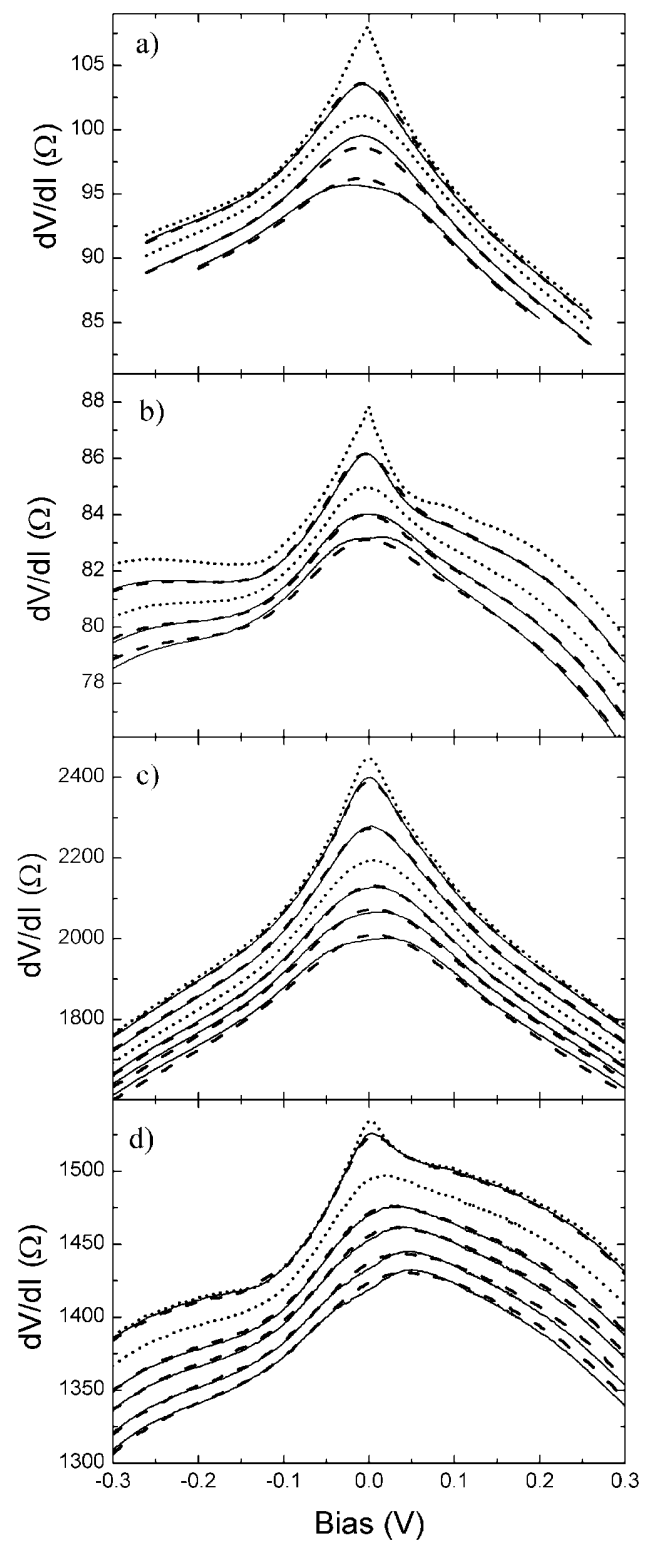

Fig. 2

Fig. $1-\mathrm{d} V / \mathrm{d} I$ vs. bias of (a) sample A in the AP state at $T=5,136,190,230$ and $267 \mathrm{~K}$, (b) sample A in the P state at $T=5,90,139,173$ and $207 \mathrm{~K}$, (c) sample B in the AP state at $T=5,72,145$, $190,222,251$ and $283 \mathrm{~K}$, and (d) sample B in the P state at $T=5,71,147,190,230,252$ and $275 \mathrm{~K}$. The curves in (d) have been shifted along the vertical axis for clarity. The data at $5 \mathrm{~K}$ (dotted line) is used to generate the thermally smeared curves by integration with $\mathrm{d} f / \mathrm{d} V$ at $T_{\mathrm{sm}}$. The dashed lines are the experimental data at higher temperature and the solid lines are best fits to the data.

Fig. $2-\mathrm{d} V / \mathrm{d} I$ vs. bias for the same samples and temperatures as in fig. 1 with the second term of eq. (2a) included. The dotted lines are the experimental data at $5 \mathrm{~K}$ and at a temperature $T_{1}$ above $100 \mathrm{~K}$, used as the input for calculating curves at all other temperatures. The dashed lines are the experimental data at other temperatures, and the solid lines are the calculated curves for the same temperatures. 
where

$$
\begin{aligned}
& A(E-V, E)=\mathrm{M}(E-V, E) N_{\mathrm{L}}(E-V) N_{\mathrm{R}}(E), \\
& B(E-V, E)=\frac{\mathrm{d}}{\mathrm{d} V}\left(\mathrm{M}(E-V, E) N_{\mathrm{L}}(E-V) N_{\mathrm{R}}(E)\right) .
\end{aligned}
$$

Without any assumptions about the matrix element and the densities of states on either side, we see that at finite temperatures their product, $A(E, V)$, and its derivative, $B(E, V)$, get thermally smeared with the Fermi function derivative, and the Fermi function itself, respectively. This happens regardless whether $A(E, V)$ has its own temperature dependence or not. It is rather obvious from eq. (2a) that thermal smearing is especially important when the thermal energy $k T$ becomes comparable to or larger than the width, $\mathrm{eV}$, of the tunneling characteristic features. It is noteworthy that the FWHM of $\mathrm{d} f / \mathrm{d} V$ is $V \simeq 3.53 \mathrm{kT} / \mathrm{e}$, which corresponds to $\sim 91 \mathrm{mV}$ at $T=300 \mathrm{~K}$.

One of the frequently used approaches for thermal smearing analysis is to neglect the second term, i.e. $B=0$, assuming that it usually provides only a small correction. If we use this assumption,

$$
\frac{\mathrm{d} I}{\mathrm{~d} V}=\int_{-\infty}^{+\infty} \mathrm{M}(E-V, E) N_{\mathrm{L}}(E-V) N_{\mathrm{R}}(E) \frac{\mathrm{d} f(E-V)}{\mathrm{d} V} \mathrm{~d} E,
$$

and, hence, thermal smearing at a finite $T$ is only due to $\mathrm{d} f(E-V) /\left.\mathrm{d} V\right|_{T}$.

In this case, at $T=0$, when $\mathrm{d} f / \mathrm{d} V$ is a delta-function, the differential conductivity, $\mathrm{d} I / \mathrm{d} V$, is a direct measure of the product of the tunneling matrix element and the two densities of states (for $E=V$ ),

$$
\begin{aligned}
\left.\frac{\mathrm{d} I}{\mathrm{~d} V}\right|_{T=0} & =\left.\int_{-\infty}^{+\infty} \mathrm{M}(E-V, E) N_{\mathrm{L}}(E-V) N_{\mathrm{R}}(E) \frac{\mathrm{d} f(E-V)}{\mathrm{d} V}\right|_{T=0} \mathrm{~d} E \\
& =\mathrm{M}(0, V) N_{\mathrm{L}}(0) N_{\mathrm{R}}(V) .
\end{aligned}
$$

We can use the low-temperature $(T=5 \mathrm{~K})$ experimental $\mathrm{d} I / \mathrm{d} V$ data as $T=0$ data, since the thermal smearing at this temperature is still very small compared to the features of $\mathrm{d} I / \mathrm{d} V$. Thus, from eq. (5) we find $\mathrm{M}(0, V) N_{\mathrm{L}}(0) N_{\mathrm{R}}(V)=\left.\frac{\mathrm{d} I}{\mathrm{~d} V}\right|_{T=5 \mathrm{~K}} ^{\exp }$. We calculate $\mathrm{d} I / \mathrm{d} V$ at finite temperatures by integrating this expression with $\mathrm{d} f / \mathrm{d} V$, evaluated at the temperature of interest $\left(T_{\text {smear }}\right)$. Since the differential conductivity data is a function of one variable, we use an approximation for the product of the matrix element and the densities of states, such that

$$
\mathrm{M}(E-V, E) N_{\mathrm{L}}(E-V) N_{\mathrm{R}}(E) \simeq \mathrm{M}(0, E) N_{\mathrm{L}}(0) N_{\mathrm{R}}(E) .
$$

While this approach correctly introduces the smearing effect of temperature, it might not estimate correctly some bias-dependent contributions such as considered in ref. [19]. The effect of the spin polarization being reduced as the temperature is increased can slightly rescale the $\mathrm{d} I / \mathrm{d} V$ data. In fitting the experimental data with the results of analytic curves we hence allow for a small bias-independent shift of the data along the resistance axis.

The solid lines in fig. 1 are fits based on thermal smearing as described above. Good agreement between data and fits can be obtained for both states at all temperatures, although the fits are somewhat better in the AP state. The best fit is always obtained for a $T_{\text {smear }}$ that is proportional to, but consistently higher than, the experimental temperature $\left(T_{\exp }\right)$. This is shown in fig. 3, where we plot the optimum smearing temperature $v s . T_{\exp }$. The direct proportionality between $T_{\text {smear }}$ and $T_{\text {exp }}$ strongly suggests that thermal smearing plays an important role. Linear fits to the data in fig. 3 suggest a $T_{\text {smear }}$ to $T_{\exp }$ ratio of $1.9(2.0)$ for sample $\mathrm{A}$ in the $\mathrm{P}$ (AP) 


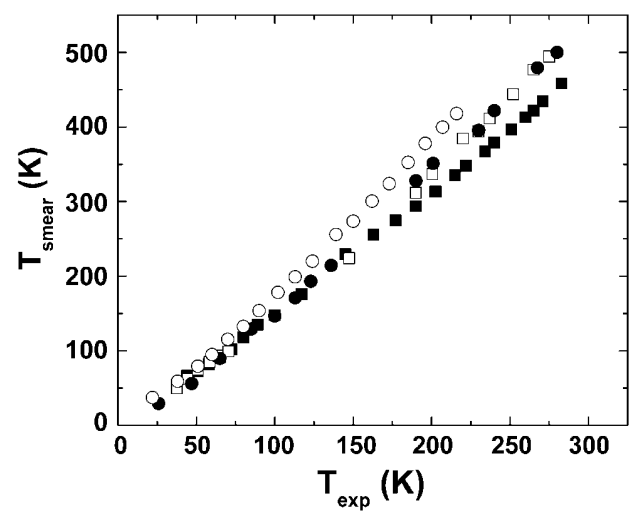

Fig. $3-T_{\text {smear }}$ vs. $T_{\text {exp }}$ for sample A in the $\mathrm{AP}(\circ)$ and $\mathrm{P}(\bullet)$ states and sample B in the AP ( $\left.\square\right)$ and $\mathrm{P}(\mathbf{\square})$ states.

state and 1.8 (1.6) for sample B in the $\mathrm{P}$ (AP) state. There is hence a tendency for a somewhat larger $T_{\text {smear }}$ for the $\mathrm{P}$ state and for samples with lower RA. The fact that $T_{\text {smear }} / T_{\exp }>1$ indicates that although the temperature dependence of tunneling characteristics is inevitably affected by thermal smearing, our very simple consideration underestimates thermal smearing effect, or a somewhat higher effective temperature of electrons should be used.

It is noteworthy that thermal smearing also correctly reproduces the shift of the maximum of $\mathrm{d} V / \mathrm{d} I$ in fig. $1 \mathrm{~d}$ and the absence thereof in fig. 1a-c. The shift is a result of the general asymmetry of the low- $T$ data in fig. $1 \mathrm{~d}$, i.e. the higher values at positive bias effectively "pull" the maximum of $\mathrm{d} V / \mathrm{d} I$ over to the positive bias side as the averaging bias window increases with $T$. The gradual disappearance of the local minimum at about $-0.12 \mathrm{~V}$ in fig. $1 \mathrm{~b}$ is also correctly reproduced by the fits.

One of the reasons of discrepancy between $T_{\text {smear }}$ and $T_{\exp }$ is that by neglecting the second term of eq. (2a) we underestimate the thermal smearing. Let us now consider the case when both terms in eq. (2a) are kept. While the technical procedure in this case is somewhat more complicated, the underlying physics is the same: we first extract two unknowns $A$ and $B$ in eq. (2b) by using two experimental curves, at two different temperatures, and solving the resulting two equations. Then, we find $\mathrm{d} I / \mathrm{d} V$ at any finite temperature by thermally smearing $A$ and $B$ with $\mathrm{d} f / \mathrm{d} V$ and $f$, respectively, evaluated at that temperature.

We again use an approximation set by eq. (6), and the fact that at $T=0$, where $\mathrm{d} f / \mathrm{d} V$ is a delta-function of $E-V$. From eq. (2b) at two temperatures, $T=0$ and $T=T_{1}$, we get two equations:

$$
\begin{aligned}
\left.\frac{\mathrm{d} I}{\mathrm{~d} V}\right|_{T=0} & =A(V)+\left.\int_{-\infty}^{+\infty} B(E)[f(E-V)-f(E)]\right|_{T=0} \mathrm{~d} E \\
\left.\frac{\mathrm{d} I}{\mathrm{~d} V}\right|_{T=T_{1}} & =\left.\int_{-\infty}^{+\infty} A(E) \frac{\mathrm{d} f(E-V)}{\mathrm{d} V}\right|_{T=T_{1}} \mathrm{~d} E+\left.\int_{-\infty}^{+\infty} B(E)[f(E-V)-f(E)]\right|_{T=T_{1}} \mathrm{~d} E .
\end{aligned}
$$

Substituting the experimental data $\mathrm{d} I /\left.\mathrm{d} V\right|_{T=5 \mathrm{~K}}$ and $\mathrm{d} I /\left.\mathrm{d} V\right|_{T=T_{1}}$, where $T_{1}$ is high enough to show the effect of thermal smearing, into the left side of eqs. (7) and solving for $A$ and $B$, we then plug them back into eq. (2b), and generate $\mathrm{d} I / \mathrm{d} V$ at any $T$.

We again allow for a shift along the vertical axis, the only adjustable parameter in this procedure. The calculated curves are in a good agreement with the experimental data (fig. 2) 
at the same $T_{\exp }$. This implies conclusively that thermal smearing is the prevailing factor for the temperature dependence of the tunneling current in MTJs, and it cannot be neglected.

The nature of the shift and its temperature dependence can be analyzed by looking at the spin polarization reduction with increasing temperature, following an approach described in ref. [10]. However, some shift can be due to the additional resistance in series inside the sample package, especially for the low-resistance sample. This additional resistance is small and negligible for the high resistance junctions.

Other possible sources of thermal effects include inelastic phonon- and magnon-assisted tunneling in the electrodes $[7,23]$, inelastic tunneling via intermediate states in the barrier $[13,24]$, thermal changes in the shape and the dielectric constant of the barrier, thermal changes of the two densities of states, etc. All these would contribute a temperature dependence of $\mathrm{M}(E-V, E) N_{\mathrm{L}}(E-V) N_{\mathrm{R}}(E)$ that is neglected in the consideration presented here. It is possible that including the temperature dependence of the various inelastic tunneling contributions would make the agreement of the fits with the experimental data perfect. Alternatively, one might speculate that the electron energy distribution at the interface is described by an effective temperature $T^{*}>T_{\exp }$ as a result of the constant influx of hot electrons, similar to what has been suggested for superconducting tunneling [25]. All these are beyond the scope of this paper.

In conclusion, we demonstrate the striking effect thermal smearing has on the MTJ tunneling curves. This effect is much more pronounced than it was previously believed, and very noticeable in the strong zero-bias anomaly generally observed in MTJs. In all samples investigated, the experimental data can be fit with only one adjustable parameter, a shift along the $\mathrm{d} V / \mathrm{d} I$ axis. In view of our results, it is obvious that any attempt to extract material properties from the temperature-dependent TMR must incorporate the large effects of thermal smearing.

We thank Dr. R. R. Ramazashvili and Prof. L. J. Sham for fruitful discussions, and Prof. Dr. G. Guntherodt for careful reading of the manuscript and constructive criticism. The work at UC San Diego was supported by DARPA, ONR and the US-DOE. The work at Motorola Labs was partially funded by DARPA.

\section{REFERENCES}

[1] Julliere M., Phys. Lett. A, 54 (1975) 225.

[2] Wolf S. A. and Treger D., IEEE Trans. Magn., 36 (2000) 2748.

[3] Miyazaki T. and Tezuka N., J. Magn. E G Magn. Mater., 139 (1995) L231.

[4] Moodera J. S., Kinder L. R., Wong T. M. and Meservey R., Phys. Rev. Lett., 74 (1995) 3273.

[5] Tehrani S. et al., IEEE Trans. Mag., 35 (1999) 2814; 36 (2000) 2752.

[6] Zhang J., Data Storage, 5 (1998) 31.

[7] Zhang S., Levy P. M., Marley A. C. and Parkin S. S. P., Phys. Rev. Lett., 79 (1997) 3744.

[8] Han X.-F. et al., Phys. Rev. B, 63 (2001) 224404.

[9] MacDonald A. H., Jungwirth T. and Kasner M., Phys. Rev. Lett., 81 (1998) 705.

[10] Shang C. H., Nowak J., Jansen R. and Moodera J. S., Phys. Rev. B, 58 (1998) R2917.

[11] Li S., de Groot C. and Moodera J. H., Appl. Phys. Lett., 77 (2000) 3630.

[12] Hagler T., Kinder R. and Bayreuther G., J. Appl. Phys., 89 (2001) 7570.

[13] Zhang J. and White R. M., J. Appl. Phys., 83 (1998) 6512.

[14] Guinea F., Phys. Rev. B, 58 (1998) 9212.

[15] Inoue J. and Maekawa S., J. Magn. ES Magn. Mater., 198 (1999) 167. 
[16] Tezuka N., Oogane M. and Miyazaki T., J. Magn. ES Magn. Mater., 198 (1999) 149.

[17] Davis A. H., Maclaren J. M. and LeClair P., J. Appl. Phys., 89 (2001) 7567.

[18] Giaever I., Hart J. H. R. and Megerle K., Phys. Rev., 126 (1962) 941.

[19] Simmons J. G., J. Appl. Phys., 35 (1964) 2655.

[20] Slaughter J. M. et al., JOM-e, 56 (6) 2000, http://www.tms.org/pubs/journals/Jom/0006/ Slaughter/Slaughter-0006.html.

[21] Jönsson-Akerman B. J. et al., Appl. Phys. Lett., 77 (2000) 1870; Rabson D. et al., J. Appl. Phys., 89 (2001) 2786; Åkerman J. J., Schuller I. K., Slaughter J. M. and Dave R. W., Appl. Phys. Lett., 79 (2001) 3104.

[22] Duke C. B., Tunneling in Solids (Academic Press, New York) 1969.

[23] Solymar L., Superconducting Tunneling and Applications (Wiley, New York) 1972, p. 66.

[24] Wolfram T. (Editor), Inelastic Electron Tunneling Spectroscopy, Vol. 4, Springer Ser. Solid State Sci. (Springer, New York) 1978.

[25] Gray K. E., in Nonequilibrium Superconductivity, Phonons and Kapitza Boundaries, edited by Gray K. E. (Plenum Press, New York) 1981, p. 131. 